PROCEEDINGS OF THE

AMERICAN MATHEMATICAL SOCIETY

Volume 129, Number 9, Pages 2647-2654

S 0002-9939(00)05891-3

Article electronically published on December 7, 2000

\title{
ON STATISTICAL LIMIT POINTS
}

\author{
P. KOSTYRKO, M. MAČAJ, T. ŠALÁT, AND O. STRAUCH \\ (Communicated by David Preiss)
}

\begin{abstract}
The set of all statistical limit points of a given sequence $x_{n}$ is characterized as an $F_{\sigma}$-set. It is also characterized in terms of discontinuity points of distribution functions of $x_{n}$.
\end{abstract}

\section{INTRODUCTION}

Following the concept of a statistically convergent sequence, J. A. Fridy [Fr93] introduced the notion of a statistical limit point of a given sequence $x_{n}, n=1,2, \ldots$, of real numbers: A real number $x$ is said to be a statistical limit point of the sequence $x_{n}$ if there exists a subsequence $x_{k_{n}}, n=1,2, \ldots$, such that $\lim _{n \rightarrow \infty} x_{k_{n}}=x$ and the set of indices $k_{n}$ has a positive upper asymptotic density (see Definitions and Notations). Fridy studied the set $\Lambda\left(x_{n}\right)$ of all such points. He has shown that this set need not be closed or open in $\mathbb{R}$.

In section 10 of the paper we prove that the set $\Lambda\left(x_{n}\right)$ is an $F_{\sigma}$-set in $\mathbb{R}$ for an arbitrary sequence $x_{n}$ and vice-versa for any given $F_{\sigma}$-set $X$ there exists a sequence $x_{n}$ such that $X=\Lambda\left(x_{n}\right)$.

In section 2 we prove that the set $\Lambda\left(x_{n}\right)$ coincides with the set of all discontinuity points of distribution functions of $x_{n}$. This leads to some sufficient conditions for $\Lambda\left(x_{n}\right)=\emptyset$.

Finally, in section 3 we compute $\Lambda\left(x_{n}\right)$ using an explicit form of the set $G\left(x_{n}\right)$ of all distribution functions of $x_{n}$ for some special $x_{n}$.

\section{DEFINITIONS AND NOTATIONS}

If $A \subset \mathbb{N}$, then we denote by $|A|$ the cardinality of $A$ and $A(n)=|\{k \leq n ; k \in A\}|$. The numbers

$$
\underline{d}(A)=\liminf _{n \rightarrow \infty} \frac{A(n)}{n}, \quad \bar{d}(A)=\limsup _{n \rightarrow \infty} \frac{A(n)}{n}
$$

are called the lower and upper asymptotic density of $A$, respectively. If there exists

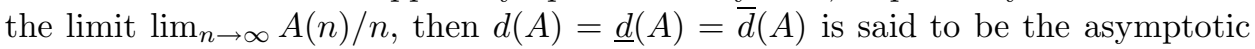
density of $A$ (cf. [HR66, xix]).

Received by the editors December 12, 1998 and, in revised form, January 10, 2000

2000 Mathematics Subject Classification. Primary 40A05, 11K31, 11 B05.

Key words and phrases. Statistically convergent sequence, statistical limit point, asymptotic density, distribution function.

This work was supported in part by grants from VEGA No. 2/5123/98 and No. 1/4323/97. 
It is easy to see that if $A$ is ordered to the increasing sequence $k_{1}<k_{2}<\ldots$, then

$$
\underline{d}(A)=\liminf _{n \rightarrow \infty} \frac{n}{k_{n}}, \quad \bar{d}(A)=\limsup _{n \rightarrow \infty} \frac{n}{k_{n}}
$$

and we write $\underline{d}(A)=\underline{d}\left(k_{n}\right)$ and $\bar{d}(A)=\bar{d}\left(k_{n}\right)$.

In the following $x_{n}, n=1,2, \ldots$, is an infinite sequence of real numbers.

The main object of the paper is the set $\Lambda\left(x_{n}\right)$ defined as

$$
\Lambda\left(x_{n}\right)=\left\{\alpha \in \mathbb{R} ; \exists\left(k_{1}<k_{2}<\ldots\right) \lim _{n \rightarrow \infty} x_{k_{n}}=\alpha, \bar{d}\left(k_{n}\right)>0\right\} .
$$

In section 2 we use the set $G\left(x_{n}\right)$ of all distribution functions of the sequence $x_{n}$, where $g:(-\infty, \infty) \rightarrow[0,1]$ is said to be a distribution function of the sequence $x_{n}$ if there is a sequence $N_{1}<N_{2}<\ldots$ of positive integers such that

$$
\lim _{k \rightarrow \infty} \frac{1}{N_{k}} \sum_{n=1}^{N_{k}} c_{(-\infty, x)}\left(x_{n}\right)=g(x)
$$

for every continuity point $x$ of $g$. Here $c_{(-\infty, x)}(t)$ is the characteristic function of the interval $(-\infty, x)$.

$x \bmod 1$ is the fractional part of $x$ and for reduced sequence $x_{n}$ mod 1 we take the restrictions of distribution functions $g(x) \in G\left(x_{n} \bmod 1\right)$ to the interval $[0,1]$.

For singleton $G\left(x_{n}\right)=\{g\}, g$ is called the asymptotic distribution function of $x_{n}$.

The sequence $x_{n}$ statistically converges to $\alpha$ if $d\left(\left\{n \in \mathbb{N} ;\left|x_{n}-\alpha\right| \geq \varepsilon\right\}\right)=0$ for any $\varepsilon>0$.

Finally, $X \bmod 1=\{x \bmod 1 ; x \in X\}$ and $X+k=\{x+k ; x \in X\}$ for a set $X$ of real numbers.

\section{Topological Structure of $\Lambda\left(x_{n}\right)$}

As we already mentioned the set $\Lambda\left(x_{n}\right)$ need not belong to the zero Borel class in $\mathbb{R}$. Thus, the following result is the best possible in this direction.

Theorem 1.1. For every sequence $x_{n}$, the set $\Lambda\left(x_{n}\right)$ is an $F_{\sigma}$-set in $\mathbb{R}$.

Proof. For any $0<t \leq 1$ we denote

$$
\Lambda\left(x_{n}, t\right)=\left\{\alpha \in \mathbb{R} ; \exists\left(k_{1}<k_{2}<\ldots\right) \lim _{n \rightarrow \infty} x_{k_{n}}=\alpha, \bar{d}\left(k_{n}\right) \geq t\right\} .
$$

We prove that for every $0<t \leq 1$, the set $\Lambda\left(x_{n}, t\right)$ is closed in $\mathbb{R}$. This gives the theorem directly, since $\Lambda\left(x_{n}\right)=\bigcup_{j=1}^{\infty} \Lambda\left(x_{n}, 1 / j\right)$.

Assume that $\alpha_{i} \in \Lambda\left(x_{n}, t\right)$ and $\lim _{i \rightarrow \infty} \alpha_{i}=\alpha$, for $i=1,2, \ldots$. For every $\alpha_{i}$ we can select a subsequence of $x_{n}$ which converges to $\alpha_{i}$ having the set of indices $K^{(i)}$ with $\bar{d}\left(K^{(i)}\right) \geq t$. Moreover, for an arbitrary given sequence $\varepsilon_{i}$ of positive numbers we can select $N_{1}<N_{2}<\ldots$ such that

$$
\frac{\left|K^{(i)} \cap\left(N_{i-1}, N_{i}\right]\right|}{N_{i}} \geq t-\varepsilon_{i}
$$

for $i=1,2, \ldots$ Putting $K=\bigcup_{i=1}^{\infty}\left(K^{(i)} \cap\left(N_{i-1}, N_{i}\right]\right)$ and assuming $\lim _{i \rightarrow \infty} \varepsilon_{i}=0$ we have $\bar{d}(K) \geq t$. Furthermore, if $K$ is ordered to increasing $k_{n}$, then $\lim _{k \rightarrow \infty} x_{k_{n}}$ $=\alpha$, because $K \subset \bigcup_{i=j}^{\infty} K^{(i)} \subset\left\{n \in \mathbb{N} ;\left|x_{n}-\alpha\right|<\varepsilon\right\}$ with the exception of finitely many terms for any $\varepsilon>0$ and sufficiently large $j=j(\varepsilon)$. 
In connection with Theorem 1.1 the question arises as to whether an arbitrary $F_{\sigma}$-set coincides with a $\Lambda\left(x_{n}\right)$ for a suitable $x_{n}$. We give the positive answer to this question.

Theorem 1.2. If $X \subset \mathbb{R}$ is an arbitrary $F_{\sigma}$-set in $\mathbb{R}$, then there exists a sequence $x_{n}$ such that $\Lambda\left(x_{n}\right)=X$.

Proof. Let $X=\bigcup_{k=1}^{\infty} X_{k}$, where $X_{k}$ are nonempty closed sets. For every $k=$ $1,2, \ldots$ select $x_{k, i} \in X_{k}, i=1,2, \ldots$, such that the set of all usual limit points of the sequence $x_{k, i}$ coincide with $X_{k}$. Decompose $\mathbb{N}=\bigcup_{k=1}^{\infty} A_{k}$ on pairwise disjoint sets $A_{k}$ having positive asymptotic density $d\left(A_{k}\right)$ and such that $d\left(\mathbb{N} \backslash \bigcup_{i=1}^{k} A_{i}\right) \rightarrow$ 0 for $k \rightarrow \infty$ (cf. [Fr93. Ex. 3]). Finally, for every $k=1,2, \ldots$, decompose $A_{k}=\bigcup_{i=1}^{\infty} B_{k, i}$ on pairwise disjoint sets $B_{k, i}$ having $\bar{d}\left(B_{k, i}\right)=d\left(A_{k}\right)$ for every $i=$ $1,2, \ldots$ (First, decompose $A_{k}=B_{k, 1} \cup B_{k, 1}^{*}$ such that $d\left(A_{k}\right)=\bar{d}\left(B_{k, 1}\right)=\bar{d}\left(B_{k, 1}^{*}\right)$. Then decompose $B_{k, 1}^{*}=B_{k, 2} \cup B_{k, 2}^{*}$ such that $\bar{d}\left(B_{k, 1}^{*}\right)=\bar{d}\left(B_{k, 2}\right)=\bar{d}\left(B_{k, 2}^{*}\right)$, etc. $)$

For a searching sequence $x_{n}$, for every $n \in B_{k, i}$, we define $x_{n}$ as $x_{n}=$ constant $=$ $x_{k, i}$. Then we have:

$1^{\circ}$. If $x_{n_{j}} \rightarrow \alpha$ for $j \rightarrow \infty$ and $\alpha \notin X$, then for every $k$ the set $\bigcup_{i=1}^{k} A_{i}$ contains only finitely many $n_{j}$ and thus $d\left(n_{j}\right)=0$.

$2^{\circ}$. If $\alpha \in X_{k}, 0<\varepsilon<d\left(A_{k}\right)$, and $x_{k, i_{j}} \rightarrow \alpha$ for $j \rightarrow \infty$, we can select $N_{1}<N_{2}<\ldots$ such that

$$
\frac{\left|B_{k, i_{j}} \cap\left(N_{j-1}, N_{j}\right]\right|}{N_{j}} \geq d\left(A_{k}\right)-\varepsilon
$$

Then $x_{n}$ over $n \in \bigcup_{j=1}^{\infty} B_{k, i_{j}} \cap\left(N_{j-1}, N_{j}\right]$ converges to $\alpha$ and

$$
d\left(\bigcup_{j=1}^{\infty} B_{k, i_{j}} \cap\left(N_{j-1}, N_{j}\right]\right) \geq d\left(A_{k}\right)-\varepsilon .
$$

\section{Points of $\Lambda\left(x_{n}\right)$ AS Discontinuity points of $g \in G\left(x_{n}\right)$}

I. J. Schoenberg [Sch59] noted that the sequence $x_{n}$ is statistically convergent to $\alpha$ if and only if it admits asymptotic distribution function $c_{[\alpha, \infty)}(x)$. This motivates the following theorem.

Theorem 2.1. For every sequence $x_{n}$ we have

$$
\Lambda\left(x_{n}\right)=\left\{\alpha \in \mathbb{R} ; \exists\left(g(x) \in G\left(x_{n}\right)\right) g(x) \text { is discontinuous at } \alpha\right\} .
$$

Proof. $1^{\circ}$. Let $\alpha$ be a discontinuity point of $g(x) \in G\left(x_{n}\right)$ with a jump of size $h$. Let $\alpha_{i}$ and $\beta_{i}$ be two sequences satisfying $\beta_{i}-\alpha_{i} \rightarrow 0$ as $i \rightarrow \infty, \alpha_{i}<\alpha<\beta_{i}$ and $\alpha_{i}$ and $\beta_{i}$ are continuity points of $g(x)$ for every $i$. From $N_{k}$ (using the definition of $g(x))$ we can select a subsequence $N_{k_{i}}$ such that

$$
\frac{1}{N_{k_{i}}} \sum_{n=N_{k_{i-1}}+1}^{N_{k_{i}}} c_{\left[\alpha_{i}, \beta_{i}\right)}\left(x_{n}\right)>h-\varepsilon
$$

for some $\varepsilon \in(0, h)$. Ordering elements of $\bigcup_{i=1}^{\infty}\left\{n \in \mathbb{N}, N_{k_{i-1}}<n \leq N_{k_{i}}\right\}$ to an increasing sequence $n_{i}$, we then have $x_{n_{i}} \rightarrow \alpha$ and $\bar{d}\left(n_{i}\right) \geq h-\varepsilon$. 
$2^{\circ}$. Assume that $x_{n_{i}} \rightarrow \alpha$ for $i \rightarrow \infty, \bar{d}\left(n_{i}\right)=h>0$, and $\varepsilon \in(0, h)$. Then there exists a sequence $N_{k}$ such that, for every $k=1,2, \ldots$,

$$
\frac{\left|\left\{i \in \mathbb{N} ; n_{i} \leq N_{k}\right\}\right|}{N_{k}} \geq h-\varepsilon
$$

By Helly selection principle from $N_{k}$ we can select a subsequence $N_{k_{i}}$ such that

$$
\lim _{i \rightarrow \infty} \frac{1}{N_{k_{i}}} \sum_{n=1}^{N_{k_{i}}} c_{(-\infty, x)}\left(x_{n}\right)=g(x)
$$

for every point $x$ of continuity of $g(x)$. Clearly, $g(x)$ has at $\alpha$ a jump of size $\geq h-\varepsilon>0$ and $g(x) \in G\left(x_{n}\right)$.

Using Theorem 2.1 some results from the theory of uniform distribution can be transcribed for $\Lambda\left(x_{n}\right)$. We can now state an analogue of the well-known WienerSchoenberg theorem and Van der Corput difference theorem, but first we begin with a technical result.

Proposition 2.2. (i) For any sequence $x_{n}, \Lambda\left(x_{n} \bmod 1\right)=\emptyset \Longrightarrow \Lambda\left(x_{n}\right)=\emptyset$.

(ii) For bounded sequence $x_{n}$, the sets $\Lambda\left(x_{n} \bmod 1\right)$ and $\Lambda\left(x_{n}\right) \bmod 1$ can differ only in 0 or 1 and if $0 \in \Lambda\left(x_{n}\right) \bmod 1$, then $0 \in \Lambda\left(x_{n} \bmod 1\right)$ or $1 \in \Lambda\left(x_{n} \bmod 1\right)$.

Proof. The implication $x_{k_{n}} \rightarrow \alpha \Longrightarrow x_{k_{n}} \bmod 1 \rightarrow \alpha \bmod 1$ does not hold only for integer $\alpha$ and $x_{k_{n}}<\alpha$.

Theorem 2.3. Let $x_{n}$ be a sequence of real numbers, and let

$$
\omega_{h}=\limsup _{N \rightarrow \infty}\left|\frac{1}{N} \sum_{n=1}^{N} e^{2 \pi i h x_{n}}\right|^{2}
$$

for $h=1,2, \ldots$ Then

$$
\lim _{H \rightarrow \infty} \frac{1}{H} \sum_{h=1}^{H} \omega_{h}=0 \Longrightarrow \Lambda\left(x_{n}\right)=\emptyset .
$$

Proof. It suffices to prove

$$
\lim _{H \rightarrow \infty} \frac{1}{H} \sum_{h=1}^{H} \omega_{h}=0 \Longrightarrow \forall\left(g(x) \in G\left(x_{n} \bmod 1\right)\right) g(x) \text { is continuous }
$$

since (by Theorem 2.1) the right-hand side implies $\Lambda\left(x_{n} \bmod 1\right)=\emptyset$ which (by Proposition 2.2 (i)) gives $\Lambda\left(x_{n}\right)=\emptyset$. For a singleton $G\left(x_{n} \bmod 1\right)=\{g(x)\}$ the Wiener-Schoenberg theorem states

$$
\lim _{H \rightarrow \infty} \frac{1}{H} \sum_{h=1}^{H} \omega_{h}=0 \Longleftrightarrow g(x) \text { is continuous. }
$$

Next, we adapt the proof which appeared in [KN74, pp. 55-56].

For $g(x) \in G\left(x_{n} \bmod 1\right)$ we select $N_{k}, k=1,2, \ldots$, such that

$$
\lim _{k \rightarrow \infty} \frac{1}{N_{k}} \sum_{n=1}^{N_{k}} c_{[\alpha, x)}\left(x_{n} \bmod 1\right)=g(x)
$$


for every point $x \in[0,1]$ of continuity of $g(x)$. Applying Helly-Bray lemma we compute $\omega_{h}(g)$ as

$$
\begin{aligned}
\omega_{h}(g) & =\lim _{k \rightarrow \infty}\left|\frac{1}{N_{k}} \sum_{n=1}^{N_{k}} e^{2 \pi i h x_{n}}\right|^{2}=\lim _{k \rightarrow \infty} \frac{1}{N_{k}^{2}} \sum_{m, n=1}^{N_{k}} e^{2 \pi i h\left(x_{m}-x_{n}\right)} \\
& =\int_{0}^{1} \int_{0}^{1} e^{2 \pi i h(x-y)} \mathrm{d} g(x) \mathrm{d} g(y) .
\end{aligned}
$$

Next, for every $(x, y) \in[0,1]^{2}$, the sequence $\frac{1}{H} \sum_{h=1}^{H} e^{2 \pi i h(x-y)}, H=1,2, \ldots$, converges and

$$
\lim _{H \rightarrow \infty} \frac{1}{H} \sum_{h=1}^{H} e^{2 \pi i h(x-y)}= \begin{cases}1 & \text { if } x-y \in \mathbb{Z}, \\ 0 & \text { otherwise }\end{cases}
$$

Thus by Lebesgue theorem of dominated convergence we have

$$
\begin{aligned}
\lim _{H \rightarrow \infty} \frac{1}{H} \sum_{h=1}^{H} \omega_{h}(g) & =\int_{0}^{1} \int_{0}^{1}\left(\lim _{H \rightarrow \infty} \frac{1}{H} \sum_{h=1}^{H} e^{2 \pi i h(x-y)}\right) \mathrm{d} g(x) \mathrm{d} g(y) \\
& =\iint_{X} 1 \cdot \mathrm{d} g(x) \mathrm{d} g(y),
\end{aligned}
$$

where

$$
X=\left\{(x, y) \in[0,1]^{2} ; x-y \in \mathbb{Z}\right\} .
$$

Since $X$ is a null set, then for continuous $g(x)$, the integral (1) equals zero. If $g(x)$ has at $x_{0} \in[\alpha, \beta]$ a jump, then (1) has a lower bound $\left(g\left(x_{0}+0\right)-g\left(x_{0}-0\right)\right)^{2}$. In all cases we have $\omega_{h} \geq \omega_{h}(g)$ and thus the proof is finished.

Note that, for every $h=1,2, \ldots$, there exists $g_{h}(x) \in G\left(x_{n}\right)$ such that $\omega_{h}=$ $\omega_{h}\left(g_{h}\right)$, but the $\lim \sup _{H \rightarrow \infty} \frac{1}{H} \sum_{h=1}^{H} \omega_{h}\left(g_{h}\right)>0$ need not imply

$$
\lim _{H \rightarrow \infty} \frac{1}{H} \sum_{h=1}^{H} \omega_{h}(g)>0
$$

for some $g(x) \in G\left(x_{n}\right)$.

Theorem 2.4. Let $x_{n}$ be a sequence of real numbers. If for every $k=1,2, \ldots$ the difference sequence $x_{n+k}-x_{n}, n=1,2, \ldots$, has $\Lambda\left(x_{n+k}-x_{n}\right)=\emptyset$, then $\Lambda\left(x_{n}\right)=\emptyset$.

Proof. Van der Corput difference theorem (cf. [KN74, Th. 3.1, p. 26]) in the form [St97, Th. 8] shows that if $G\left(\left(x_{n+k}-x_{n}\right) \bmod 1\right), k=1,2, \ldots$, contains only continuous distribution functions, then the same holds for $G\left(x_{n} \bmod 1\right)$. Thus we have the implication $\Lambda\left(\left(x_{n+k}-x_{n}\right) \bmod 1\right)=\emptyset, k=1,2, \cdots \Longrightarrow \Lambda\left(x_{n} \bmod 1\right)=\emptyset$.

For the general case the referee suggested the following simple proof: Assume that $x_{n}(n \in A) \rightarrow \alpha$ and $\bar{d}(A)>0$. Then there exists $k$ such that $\bar{d}(A \cap A+k)>0$ and moreover $x_{n+k}-x_{n}(n+k \in A \cap A+k) \rightarrow 0$.

In the proof of Theorem 1.2 we have constructed, for an arbitrary $F_{\sigma}$-set $X$, a sequence $x_{n}$ such that $\Lambda\left(x_{n}\right)=X$, but this $x_{n}$ is not one-to-one. The implication $\lim _{n \rightarrow \infty} x_{n}-y_{n}=0 \Longrightarrow \Lambda\left(x_{n}\right)=\Lambda\left(y_{n}\right)$ gives change $x_{n}$ to a one-to-one $y_{n}$. Inspired by [KN74, Ex. 2.11, .23] we can obtain the following generalization. 
Theorem 2.5. For any two sequences $x_{n}$ and $y_{n}$ we have

$$
\lim _{N \rightarrow \infty} \frac{1}{N} \sum_{n=1}^{N}\left|x_{n}-y_{n}\right|=0 \Longrightarrow \Lambda\left(x_{n}\right)=\Lambda\left(y_{n}\right) .
$$

Proof. For $x_{n}, y_{n} \in[0,1]\left[\right.$ St97, Th. 7] gives $\lim _{N \rightarrow \infty} N^{-1} \sum_{n=1}^{N}\left|x_{n}-y_{n}\right|=0 \Longrightarrow$ $G\left(x_{n}\right)=G\left(y_{n}\right)$ and thus $\Lambda\left(x_{n}\right)=\Lambda\left(y_{n}\right)$ applying Theorem [2.1]

For general $x_{n}, y_{n}$ the referee suggested: The limit $N^{-1} \sum_{n=1}^{N}\left|x_{n}-y_{n}\right| \rightarrow 0$ implies $\bar{d}\left(\left\{n \in \mathbb{N} ;\left|x_{n}-y_{n}\right| \geq \varepsilon\right\}\right)=0$ for every $\varepsilon>0$. Thus there exists $B \subset \mathbb{N}$ such that $d(B)=1$ and $\left|x_{n}-y_{n}\right|(n \in B) \rightarrow 0$. Now, let $x_{n}(n \in A) \rightarrow \alpha$ and $\bar{d}(A)>0$. Then $\bar{d}(A \cap B)=\bar{d}(A)>0$ and $y_{n}(n \in A \cap B) \rightarrow \alpha$.

\section{Computations of $\Lambda\left(x_{n}\right)$}

Example 3.1. If $G\left(x_{n} \bmod 1\right)=\{g(x)\}$ and $g(x)=x$, then the sequence $x_{n}$ is called uniformly distributed mod 1. Applying Theorem 2.1 and the continuity of $g(x)=x$ we find (as in [Fr93, Ex. 4]) that

$$
\Lambda\left(x_{n} \bmod 1\right)=\emptyset
$$

for every uniformly distributed sequence $x_{n} \bmod 1$. E.g. $\Lambda$-set is empty for

$n \theta \bmod 1$ with irrational $\theta$;

$n^{2} \theta+\sin (2 \pi \sqrt{n}) \bmod 1$ with irrational $\theta$ (cf. KN74, p. 31]);

$\log F_{n} \bmod 1$ with Fibonacci numbers $F_{n+1}=F_{n}+F_{n-1}, F_{1}=F_{2}=1$ (cf. [KN74, p. 31]);

$n \log \log \ldots \log n \bmod 1$ (cf. KN74, p. 24]), etc.

Example 3.2. It is well known that (cf. [KN74, p. 58])

$$
G(\log n \bmod 1)=\left\{e^{-\alpha} \frac{e^{x}-1}{e-1}+e^{-\alpha}\left(e^{\min (x, \alpha)}-1\right) ; \alpha \in[0,1]\right\} .
$$

Since all distribution functions in $G(\log n \bmod 1)$ are continuous, we have

$$
\Lambda(\log n \bmod 1)=\emptyset .
$$

Another proof follows from Theorem 2.3, because, for $x_{n}=\log n \bmod 1$, we have

$$
\omega_{h}=\frac{1}{4 \pi^{2} h^{2}+1} .
$$

More generally, for $x_{n}=t \log n \bmod 1, t \neq 0$, we have

$$
\omega_{h}=\frac{1}{4 \pi^{2} h^{2} t^{2}+1}
$$

which implies $\lim _{H \rightarrow \infty} \frac{1}{H} \sum_{h=1}^{H} \omega_{h}=0$ and thus $\Lambda(t \log n \bmod 1)=\emptyset$ for any $t \neq 0$. For computing $\omega_{h}$ we have used a method described in [Pa84, Solution 5.18, p. 281].

Example 3.3. It is proved in St95] that starting with $\log \log n \bmod 1$ all the sequences $\log \log \ldots \log n \bmod 1$ have

$$
G(\log \log \ldots \log n \bmod 1)=\left\{c_{\alpha}(x) ; \alpha \in[0,1]\right\} \cup\left\{h_{\alpha}(x) ; \alpha \in[0,1]\right\} .
$$

Here $c_{\alpha}:[0,1] \rightarrow[0,1]$ is a one-jump distribution function

$$
c_{\alpha}(x)= \begin{cases}0, & \text { if } x \in[0, \alpha), \\ 1, & \text { if } x \in(\alpha, 1] ;\end{cases}
$$


$c_{\alpha}(0)=0, c_{\alpha}(1)=1$, and $c_{\alpha}(\alpha)=0$ if $0<\alpha<1 ; h_{\alpha}:[0,1] \rightarrow[0,1]$ is constant distribution function, where $h_{\alpha}(0)=0, h_{\alpha}(1)=1$, and $h_{\alpha}(x)=\alpha$ if $x \in(0,1)$.

Applying Theorem 2.1 we have

$$
\Lambda(\log \log \ldots \log n \bmod 1)=[0,1] .
$$

Example 3.4. Let $\alpha=\frac{p}{q} \pi$, where $p$ and $q$ are positive integers and g.c.d. $(p, q)=$ 1. It is proved in [BBK95] that the sequence

$$
x_{n}=n \cos (n \cos n \alpha) \bmod 1, \quad n=1,2, \ldots,
$$

has $G\left(x_{n}\right)=\{g(x)\}$, where

$$
g(x)= \begin{cases}x & \text { if } q \text { is odd } \\ \left(1-\frac{1}{q}\right) x+\frac{1}{q} c_{0}(x) & \text { if } q \text { is even }\end{cases}
$$

and $c_{0}(x)$ is introduced in Example 3.3 for $\alpha=0$. Theorem 2.1 implies

$$
\Lambda\left(x_{n}\right)= \begin{cases}\emptyset & \text { if } q \text { is odd } \\ \{0\} & \text { if } q \text { is even }\end{cases}
$$

\section{Concluding Remarks}

In [Fr93], Fridy has also introduced the concept of statistical cluster points of a sequence $x_{n}$ and studied the set $\Gamma\left(x_{n}\right)$ of all such points. A number $\alpha$ is called the statistical cluster point of the sequence $x_{n}, n=1,2, \ldots$, provided that for every $\varepsilon>0, \bar{d}\left(\left\{n \in \mathbb{N} ;\left|x_{n}-\alpha\right|<\varepsilon\right\}\right)>0$. Fridy proved that $\Gamma\left(x_{n}\right)$ is a closed point set. Evidently, $\alpha \notin \Gamma\left(x_{n}\right)$ if and only if there exist an open interval $I$ for which $\alpha \in I$ and $g(x)=$ constant for every $x \in I$ and any $g(x) \in G\left(x_{n}\right)$. Now, let $X$ be a given nonempty closed subset of $[0,1]$ with component intervals $\bigcup_{k \in K} I_{k}=[0,1] \backslash X$. Denote $g(x)=\sum_{I_{k} \subset[0, x)} \lambda\left(I_{k}\right)+\lambda(X \cap[0, x))$, where $\lambda$ is the Lebesgue measure. We can see that $g(x)$ is a distribution function with constant value on every interval $I_{k}$ and it increases in any neighbourhood of $x \in X$. For such $g(x)$ (cf. KN74, Th. 4.3 , p. 138] there exists a sequence $x_{n} \in[0,1]$ such that $g(x)$ is an asymptotic distribution function of $x_{n}$. Thus $\Gamma\left(x_{n}\right)=X$.

G. Myerson My93 calls a sequence $x_{n} \in[0,1]$ maldistributed if for any nondegenerate interval $I \subset[0,1], \bar{d}\left(\left\{n \in \mathbb{N} ; x_{n} \in I\right\}\right)=1$. In St95] the maldistribution is characterized by $G\left(x_{n}\right) \supset\left\{c_{\alpha}(x) ; \alpha \in[0,1]\right\}$. Thus, by Theorem [2.1, $\Lambda\left(x_{n}\right)=[0,1]$ and from the definition of cluster points also, $\Gamma\left(x_{n}\right)=[0,1]$, for any maldistributed $x_{n}$. As an example of maldistributed sequences see Example 3.3.

\section{REFERENCES}

[BBK95] D. Berend, M. D. Boshernitzan, And G. Kolesnik, Distribution modulo 1 of some oscillating sequences, II. Israel J. Math. 92 (1995), 125-147. MR 96j:11105

[Fr93] J. A. Fridy, Statistical limit points. Proc. Amer. Math. Soc. 118 (1993), 1187-1192. MR 94e: 40008

[HR66] H. Halberstam and K. F. Roth, Sequences, I. Clarendon Press, Oxford, 1966. MR 35:1565

[KN74] L. Kuipers And H. Niederreiter, Uniform Distribution of Sequences. John Wiley \& Sons, New York, 1974. MR 54:7415

[My93] G. Myerson, A sampler of recent developments in the distribution of sequences. Lect. Notes Pure Appl. Math. 147 (1993), 163-190. MR 94a:11112

[Pa84] D. P. PARent, Exercises in Number Theory. Problem Books in Mathematics, SpringerVerlag, New York, Berlin, Heidelberg, Tokyo, 1984. MR 86f:11002 
[Sch59] I. J. SchoenBerg, The integrability of certain functions and related summability methods. Amer. Math. Monthly 66 (1959), 361-375. MR 21:3696

[St95] O. Strauch, Uniformly maldistributed sequences in a strict sense. Monatsh. Math. 120 (1995), 153-164. MR 96g:11095

[St97] O. Strauch, On set of distribution functions of a sequence. In: Proc. Conf. Analytic and Elementary Number Theory, In Honor of E. Hlawka's 80th Birthday, Vienna, July 18-20, 1996, Universität Wien and Universität für Bodenkultur, (W. G. Nowak and J. Schoißengeier, eds.) Vienna, 1997, 214-229.

Department of Mathematics, Comenius University, SK-842 15 Bratislava, Slovakia

E-mail address: kostyrko@fmph.uniba.sk

Department of Mathematics, Comenius University, SK-842 15 Bratislava, Slovakia

E-mail address: macaj@fmph.uniba.sk

Department of Mathematics, Comenius University, SK-842 15 Bratislava, Slovakia

Mathematical Institute of the Slovak Academy of Sciences, Štefánikova 49, SK-814 73 Bratislava, Slovakia

E-mail address: strauch@mat.savba.sk 